\title{
Novel three-dimensional imaging system may facilitate gastric endoscopic submucosal dissection procedure: an ex vivo animal study
}

다)(웅

\begin{abstract}
Authors Keiko Saito ${ }^{1}$, Tomoo Nakagawa ${ }^{1}$, Makoto Arai ${ }^{1}$, Naoya Kato ${ }^{1}$

Institutions

1 Department of Gastroenterology, Graduate School of Medicine, Chiba University, Chiba, Japan

2 Endoscopic Center, Hanoi Medical University Hospital, Hanoi, Vietnam
\end{abstract}

Naoki Akizue ${ }^{1}$, Tomoaki Matsumura' ${ }^{1}$ Daisuke Maruoka ${ }^{1}$, Kentaro Ishikawa', Dao Viet Hang ${ }^{2}$, Kenichiro Okimoto ${ }^{1}$,

submitted 6.4.2018

accepted after revision 4.9.2018

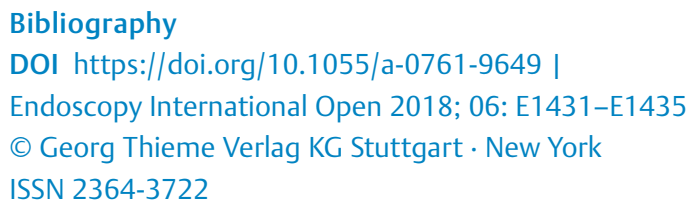

Corresponding author

Tomoaki Matsumura, MD, Department of

Gastroenterology, Graduate School of Medicine, Chiba

University, Inohana 1-8-1, Chiba-City, 260-8670, Japan

Phone: $+81-43-226-2083$

Fax: $+81-43-226-2088$

matsumura@chiba-u.jp

\section{ABSTRACT}

Background and study aims Endoscopic submucosal dissection (ESD) requires advanced skills to perform safely without complications. The current study evaluated the usefulness of a novel three-dimensional (3D) imaging system in ESD using porcine stomachs.

Methods Four endoscopists (two trainees and two experts) performed eight ESD procedures using both 3D and 2D images. The usefulness of 3D image versus 2D image was evaluated by visibility and procedure time. In addition, occurrence of eyestrain and dizziness in 3D image was assessed. Results En bloc resection was successfully achieved, without perforation, in all cases. The evaluation score in the 3D image group was better than that in the 2D image group, particularly depth perception was statistically significantly good. No significant difference was found in the working speed between the 2D and 3D image groups. Two examiners experienced eyestrain and dizziness while using the 3D image.

Conclusions All the ESD procedures were performed safely. Depth perception using the 3D image was better than with the 2D image. A novel 3D image system may facilitate ESD.

\section{Introduction}

Gastric endoscopic submucosal dissection (ESD) was developed in the late 1990s in Japan. Today, intramucosal (cT1a) differentiated lesions are resectable by ESD regardless of size after expanded indication for local treatment has been reported [1,2]. Initially, ESD was indicated for early gastric cancer and subsequently for esophageal and colorectal cancers. However, the risk for complications, such as bleeding and perforation, associated with ESD is reportedly higher than that with endoscopic mucosal resection (EMR) $[3,4]$, which is why safe performance of ESD is an important subject.

In the medical field, the experience of using three-dimensional (3D) image technology with laparoscopy was reported in the early 1990s [5]. Since then, numerous cases of laparoscopic surgery using 3D image technology have been reported, and some of them showed improvement of the procedure time, recognition accuracy, and complications [6]. In the endoscopic field, however, 3D imaging has not been put to practical use. With the evolution of ESD skills and of several endoscopic devices, it is now possible to resect more difficult lesions than before and to perform more advanced endoscopic techniques. However, the examiner must perform the endoscopic procedure in 3D space while watching the 2D image. In gastric ESD, the complication rate at a low-volume center is reportedly higher than that at a high-volume center [7]. To avoid complications, it is crucial to determine how quickly and safely one can reach the same level as an expert.

Recently, a novel 3D imaging processor that can create 3D virtual video images through conventional 2D endoscopic video images has become available [8]. In 2015, Yoshida et al. 
reported the usefulness of this system in cystoscopic surgery [9]. In the gastrointestinal tract field, we reported our experience with colonoscopy in 2017 [8]. The current study evaluated the usefulness of the 3D system in ESD using the porcine stomach.

\section{Methods}

\section{Novel 3D image system}

We used a novel 3D imaging processor (HD-3D-A; Shinko Optical, Tokyo, Japan). This processor can create a 3D image by converting the image from a conventional endoscope system. This system changes the enlargement ratio of the left and right sides of the image and creates two images and combines them, similar to the function of the human left and right eyes, respectively ( $\mathbf{F i g . 1}$ ) $[8,9]$. In this way, this system converts the $2 \mathrm{D}$ image obtained by a single lens into a $3 \mathrm{D}$ image on the $3 \mathrm{D}$ monitor. We can recognize the $3 \mathrm{D}$ image using the $3 \mathrm{D}$ glasses $[8,9]$.

\section{ESD procedure}

In this study, ESD was performed using both 3D and 2D images. Two resected porcine stomachs that are commercially available were used. These stomachs were fixed to equipment dedicated to ESD training that can be energized and approximately $20-\mathrm{mm}$ artificial lesions located in the greater curvature side of the antrum and the lesser curvature side of the body were resected using ESD. Four endoscopists performed eight ESD procedures. Two endoscopists were experts who had performed more than 300 ESD procedures and have board certification from the Japan Gastroenterological Endoscopy Society, and the other two endoscopists were trainees who had performed more than 10 gastric ESD procedures. All endoscopists performed two ESD procedures using 3D and 2D images alternately as described in -Fig. 2. A GIF Q260J endoscope (Olympus Optical Co., Ltd., Tokyo, Japan) was used. A 2.0-mm Dual knife (Olympus Optical Co., Ltd., Tokyo, Japan) was used to mark an approximately $20-\mathrm{mm}$ area, and the surrounding area was injected with Glyceol (Chugai Pharmaceutical Co., Tokyo, Japan) using a 5-mm needle. Subsequently, the Dual knife was used for precutting, then the IT knife 2 (Olympus Optical Co., Ltd., Tokyo, Japan) was used to incise around the lesion. The submucosa was dissected using the Dual knife or the IT knife 2. The electrosurgical generator used for ESD was VIO300 D (Erbe, Tubingen, Germany). The setting when precutting by Dual knife is Endocut effect 4100 W, when incising by IT knife 2 is Endocut Q E4 D3 I2, and when dissecting by Dual knife is SwiftCoag E4 $80 \mathrm{~W}$, by IT knife 2 is SwiftCoag E5 100 W.

\section{Endpoints}

The current study evaluated the en bloc resection rate, perforation rate, and usefulness of 3D image for ESD (depth perception, ease of incision, ease of dissection). Evaluation methods were assessed using a score of 1 to 5 points (1: very bad, 2: bad, 3: normal, 4: good, 5: very good), and evaluation in 2D image was set to three points. These scores were evaluated immediately after each ESD procedure. Mean speed of incision and dissection were

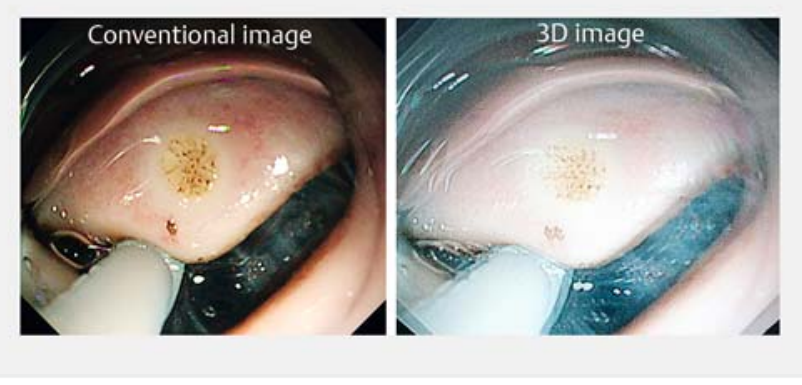

- Fig. $12 \mathrm{D}$ and 3D endoscopic images. 2D images are converted to 3D images by a novel 3D imaging processor. The 3D image can be recognized by using $3 \mathrm{D}$ glasses.

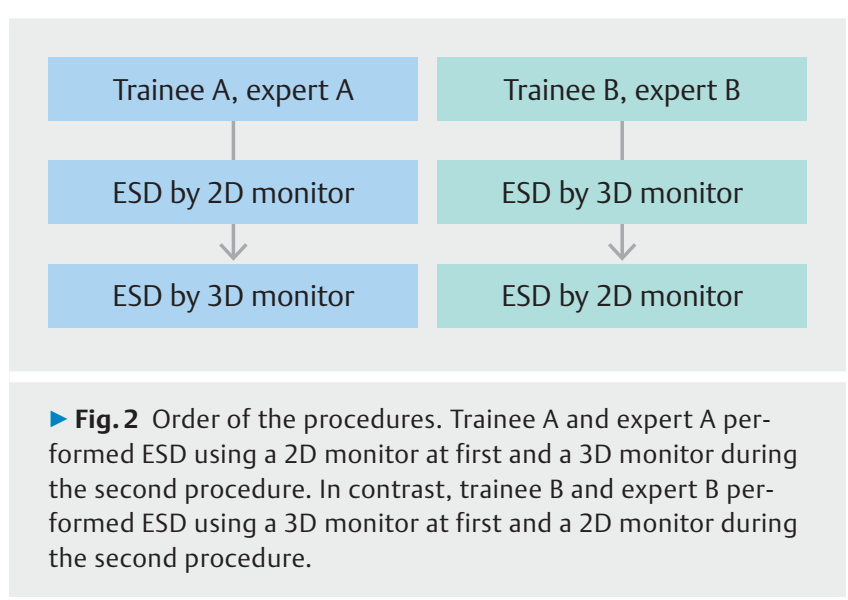

also evaluated by measuring the size of the resected specimen. In addition, presence of eyestrain and dizziness during ESD procedure was assessed by asking the endoscopists if they felt such symptoms. We chose these quality metrics with reference to previous reports on 3D surgery and modified them to evaluate the usefulness of 3D endoscopy for ESD exactly and clearly [10, 11].

\section{Statistical analysis}

Data are presented as mean \pm SD. Differences in visibility scores and procedure time between the $2 \mathrm{D}$ and $3 \mathrm{D}$ images were analyzed using the paired $t$-test. All statistical analyses were performed using SPSS 23.0 (SPSS Inc., Chicago, Illinois, United States), and a $P$ value $<0.05$ was considered to be statistically significant in 2-sided tests. This study was a pilot study. We set up realistic numbers for the pilot study without calculating a sample size.

\section{Results}

En bloc resection without perforation was successfully performed in all procedures. Details of each resected specimen are shown in $>$ Table 1. When using the 3D image, depth perception was 4.25 (95\% Cl, $3.45-5.04)$, ease of incision was $3.25(95 \% \mathrm{Cl}$, $2.45-4.04)$, and ease of dissection was $3.75(95 \% \mathrm{Cl}, 2.95-$ 
- Table 1 Details of each resected specimen.

ESD using 2D monitor

\begin{tabular}{|c|c|c|c|c|}
\hline \multirow[b]{2}{*}{ Operator } & \multicolumn{4}{|c|}{ Resected specimen } \\
\hline & Major diameter (mm) & Minor diameter $(\mathrm{mm})$ & Circumference (mm) & Area $\left(\mathrm{mm}^{2}\right)$ \\
\hline Trainee A & 34 & 29 & 198.23 & 3096.04 \\
\hline Trainee B & 30 & 20 & 158.65 & 1884 \\
\hline Expert A & 39 & 34 & 229.6 & 4163.64 \\
\hline Expert B & 28 & 23 & 160.6 & 2022.16 \\
\hline \multicolumn{5}{|c|}{ ESD using 3D monitor } \\
\hline & \multicolumn{4}{|c|}{ Resected specimen } \\
\hline Operator & Major diameter (mm) & Minor diameter $(\mathrm{mm})$ & Circumference (mm) & Area $\left(\mathrm{mm}^{2}\right)$ \\
\hline Trainee A & 17 & 16 & 103.69 & 854.08 \\
\hline Trainee B & 23 & 23 & 144.51 & 1661.06 \\
\hline Expert A & 22 & 14 & 114.5 & 967.12 \\
\hline Expert B & 23 & 15 & 120.71 & 1083.3 \\
\hline
\end{tabular}

4.54), which are good results compared with those obtained while using the 2D image. In particular, depth perception was statistically significantly good ( $\triangleright$ Fig. 3 ).

Incision speed was $42.0 \mathrm{~mm} / \mathrm{min}(95 \% \mathrm{Cl},-22.69-107.0)$ in the $3 \mathrm{D}$ image, which was faster than that obtained in the $2 \mathrm{D}$ image $(26.4 \mathrm{~mm} / \mathrm{min}$ [ $95 \% \mathrm{Cl},-14.15-67.53])$. Dissection speed was $199.2 \mathrm{~mm}^{2} / \mathrm{min}(95 \% \mathrm{Cl}, 57.54-340.59)$ in the 2D image, which was faster than the $139.2 \mathrm{~mm}^{2} / \mathrm{min}(95 \% \mathrm{Cl},-44.88-$ $323.77)$ in the 3D image, but no significant difference was found between the 2D and 3D images ( $>$ Fig. 4 ).

Eyestrain and dizziness were experienced by two examiners (trainee $A$ and expert B) when using the 3D image. Although they were able to perform the ESD procedures, they found it difficult to view the image due to the dirty screen when using the 3D image. On the other hand, the other two examiners didn't feel dizziness and eyestrain during ESD when using both 2D and 3D images.

\section{Discussion}

This is the first ESD study using a 3D image system. All ESD procedures using the 3D image system were performed safely without perforation and equipment problems. Moreover, equal or good evaluation was obtained for depth perception, ease of incision, and ease of dissection when using the 3D image. Not only experts but also trainees could perform the procedure safely. Advantages of using a 3D image might include performing the ESD procedure more safely and quickly. Incision speed was fast for the 3D monitor group, whereas dissection speed was fast for the 2D monitor group. We consider that this result might be due to the low number of cases and different resection sites. In our study, we used only two porcine stomachs for eight ESD procedures. ESD was attempted at the greater curva-

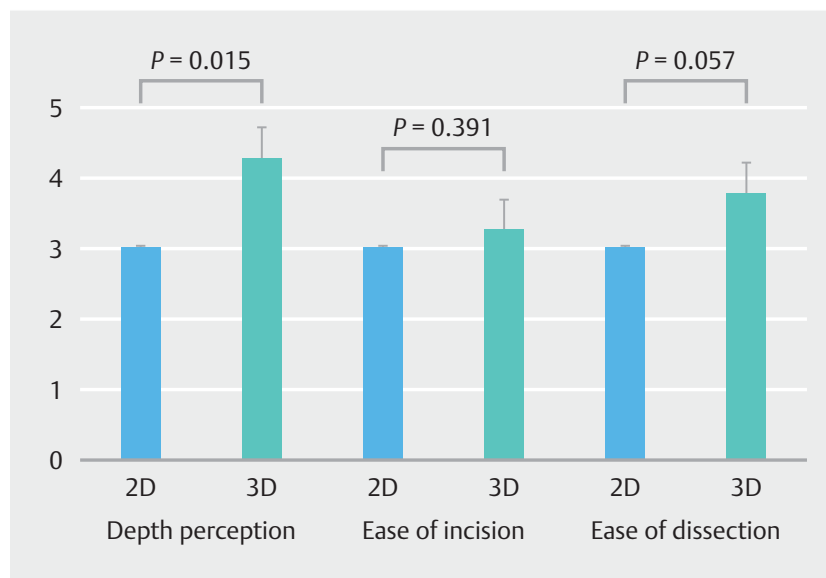

- Fig. 3 Average evaluation by operators. When using the 3D image, depth perception, ease of dissection, and ease of dissection were all good compared with results obtained using the 2D image. In particular, depth perception was significantly better with a 3D image.

ture side of the antrum as much as possible, but some ESD were performed at the lesser curvature side of the gastric body. Further studies with a large sample size are warranted to clarify this speculation.

Performance of ESD using a 3D image with the prototype 3D Olympus flexible endoscope was reported in 2017 [12]. However, that scope is a trial device and it currently cannot be used in humans. This study used a device that is connected to a conventional endoscope system and can obtain 3D images only using the 3D monitor and glasses. Therefore, the technology is an inexpensive and practical advancement in the endoscopy field. The results showed that using a 3D image during ESD 


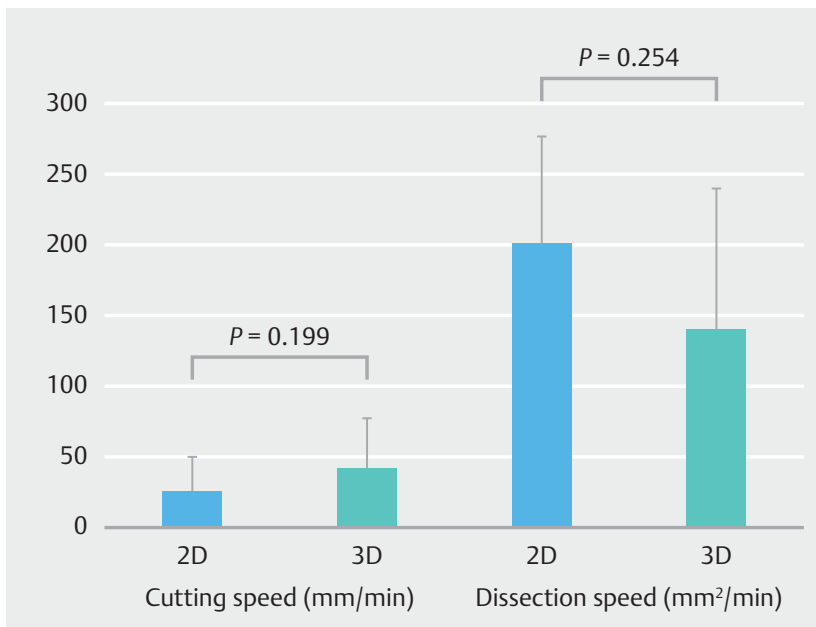

- Fig. 4 Average working speed of operators. lincision speed tended to be fast with a 3D image. Dissection speed tended to be fast with a 2D image, but no significant difference was seen between the $2 \mathrm{D}$ and $3 \mathrm{D}$ images in that measure.

can improve visibility of space compared with a 2D image, and it may be possible to safely perform ESD with favorable conditions. This novel 3D imaging processor may possibly be used not only in ESD but also in various endoscopic examinations and treatments. We can switch the image from 2D to 3D anytime. Subsequently, application of a 3D image may be made possible easily for some other procedures. We always perform the endoscopic procedure while converting the 2D image to a 3D image. Experts who have good spatial recognition ability don't struggle to convert from 2D to 3D, but for beginners, it is very difficult. Regarding $3 \mathrm{D}$ advantages, ability to grasp depth perception, endoscopic precision, and improved space orientation have been reported in some studies. Especially in ESD, those qualities are essential. When using 3D images, we can get a 3D image relatively easily to achieve proper position and depth to resect. In contrast to the positive attributes of using a 3D image, in some reports of laparoscopic surgery, use of a 3D image has resulted in eyestrain and dizziness, a finding that was consistent with this study [13]. With regard to that issue, some reports on more recent laparoscopic surgery using a 3D image showed no side effects compared with use of a 2D image [14]. The side effects may possibly be resolved by improving image resolution and experience with a 3D image. Therefore, increasing experience in using a $3 \mathrm{D}$ image is necessary.

ESD for gastric cancer is speculated to be easier than that for colorectal and esophageal cancers. However, incidence of gastric cancer is rare in the West compared with Asian countries. Therefore, Western endoscopists have difficulty in learning, and improving the learning curve is very important in the West compared with Asian countries [15]. Some studies have reported on the learning curve forf ESD, and Ebigbo et al. reported that trainees should observe those who are experts in performing ESD and perform on a porcine model before actually undertaking the procedure in patients [16]. Bhatt et al. reported on the usefulness of video-based supervision from Japanese experts for learning about and training in basic ESD [17]. Our results suggest that a 3D image system could be one of the tools that will improve the learning curve due to the ease of performing the ESD procedure.

This study has some limitations. First, only a few cases were evaluated. The sample size in this pilot study was small. Therefore, even items that did not show statistical differences may be clinically significant. In addition, in our study, the depth perception score was superior in the $3 \mathrm{D}$ group, but it is uncertain how much difference is clinically significant in this field. Second, some differences were found between the porcine model and human ESD, including the thinner layers of the human stomach wall, management of bleeding and fibrosis, and movement from respiration. To overcome these issues, more evaluations and clinical trials are required.

\section{Conclusion}

In conclusion, we were able to perform all ESD procedures safely using the 3D image system. We believe that improved depth perception using the $3 \mathrm{D}$ image could make the endoscopic procedure easier compared with using the $2 \mathrm{D}$ image. On the other hand, the usefulness of a 3D image system for gastric ESD is still uncertain because this was a pilot study using an animal and had a very small sample size. Eyestrain and dizziness noted during ESD using a 3D image need to be overcome, and the usefulness of the $3 \mathrm{D}$ image system in the endoscopic field should be further evaluated.

\section{Competing interests}

This research was conducted using grants from Chiba Foundation for Health Promotion \& Disease Prevention.

\section{References}

[1] Gotoda T, Yanagisawa A, Sasako M et al. Incidence of lymph node metastasis from early gastric cancer: estimation with a large number of cases at two large centers. Gastric Cancer 2000; 3: 219-225

[2] Hirasawa T, Gotoda T, Miyata S et al. Incidence of lymph node metastasis and the feasibility of endoscopic resection for undifferentiatedtype early gastric cancer. Gastric Cancer 2009; 12: 148-152

[3] Ohkuwa M, Hosokawa K, Boku N et al. New endoscopic treatment for intramucosal gastric tumors using an insulated-tip diathermic knife. Endoscopy 2001; 33: 221-226

[4] Shiro O, Shinji T, Iwao K et al. Advantage of endoscopic submucosal dissection compared with EMR for early gastric cancer. Gastroenterol Endosc 2006; 64: 877-883

[5] Becker H, Melzer A, Schurr MO et al. 3-D video techniques in endoscopic surgery. Endosc Surg Allied Technol 1993; 1: $40-46$

[6] Fergo C, Burcharth J, Pommergaard HC et al. Three-dimensional laparoscopy vs 2-dimensional laparoscopy with high-definition technology for abdominal surgery: a systematic review. Am J Surg 2017; 213: $159-170$

[7] Odagiri H, Yasunaga H. Complications following endoscopic submucosal dissection for gastric, esophageal, and colorectal cancer: 
a review of studies based on nationwide large-scale databases. Ann Transl Med 2017; 5: 189

[8] Matsumura T, Ishigami H, Okimoto K et al. Three-dimensional imaging system for colonoscopy. Endoscopy 2017; 49: 716-717

[9] Soichiro Y, Kazunori K, Tsuneo F et al. Novel three-dimensional image system for transurethral surgery. Int J Urol 2015; 22: 714 - 715

[10] Stine S, Mona S, Lars K et al. Three-dimensional versus two-dimensional vision in laparoscopy: a systematic review. Surg Endosc 2016; 30: $11-23$

[11] Melina CV, Liane SF, Christopher GA et al. A global assessment tool for evaluation of intraoperative laparoscopic skills. Am J Surg 2005; 190: $107-113$

[12] Kikuchi D, Kaise M, Nomura K et al. Feasibility study of the threedimensional flexible endoscope in endoscopic submucosal dissection: an ex vivo animal study. Digestion 2017; 95: 237-241
[13] Sun CC, Chiu AW, Chen KK et al. Assessment of a three-dimensional operating system with skill tests in a pelvic trainer. Urol Int 2000; 64: $154-158$

[14] Sørensen SM, Savran MM, Konge L et al. Three-dimensional versus two-dimensional vision in laparoscopy: a systematic review. Surg Endosc 2016; 30: 11 - 23

[15] Deprez PH, Bergman JJ, Meisner S et al. Current practice with endoscopic submucosal dissection in Europe: position statement from a panel of experts. Endoscopy 2010; 42: 853-858

[16] Ebigbo A, Messmann H. How can we make the learning curve of endoscopic submucosal dissection for (Western) endoscopists less steep? Endoscopy 2016; 48: $697-698$

[17] Bhatt A, Abe S, Kumaravel A et al. Video-based supervision for training of endoscopic submucosal dissection. Endoscopy 2016; 48: 711 716 